\title{
Surgical Techniques for operating on Large Adherent Cervical Nodal Metastases from Thyroid Cancer causing Severe Neck Pain and Compression Effects and encasing Major Vessels
}

\author{
${ }^{1}$ Pooja Ramakant, ${ }^{2}$ Kul R Singh, ${ }^{3}$ Chanchal Rana, ${ }^{4}$ Anand K Mishra
}

\begin{abstract}
Aim: We aim to refine and define surgical techniques for doing difficult adherent bulky cervical nodal metastases from thyroid cancer to help surgeons do dissections with better curability and lesser morbidity.
\end{abstract}

Background: Patients with thyroid cancer with large nodal metastases presenting with severe neck pain due to compression effects and encasing carotid artery and other major vessels pose a challenge to surgeons to do complete resections while preserving important structures in the neck.

Materials and methods: We define surgical planning and road map to dissect difficult bulky adherent cervical nodes encasing carotid artery and jugular vein by splaying the plane over carotid sheath by doing adventitial level dissection and dissecting medial and lateral to carotid sheath the large nodal mass adherent to adjacent structures preserving the major vessels and nerve plexus.

Conclusion: By appropriate surgical planning and meticulous dissection techniques, we can do major neck dissections with complete resections and same time preserving important structures in the neck minimizing morbidity.

Clinical significance: By doing neck dissections with complete oncological resections and saving vital structures in the neck, we aim to offer best possible chance of cure to the patient along with reduced morbidity at same time.

Keywords: Difficult neck dissections, Nodes encasing major neck vessels, Thyroid cancer.

How to cite this article: Ramakant $P$, Singh $K R$, Rana $C$, Mishra AK. Surgical Techniques for operating on Large Adherent Cervical Nodal Metastases from Thyroid Cancer causing Severe Neck Pain and Compression Effects and encasing Major Vessels. World J Endoc Surg 2016;8(3):217-219.

\footnotetext{
${ }^{1}$ Associate Professor, ${ }^{2,3}$ Assistant Professor, ${ }^{4}$ Professor

1,2,4 Department of Endocrine and Breast Surgery, King Georges' Medical University, Lucknow, Uttar Pradesh, India

${ }^{3}$ Department of Pathology, King Georges' Medical University Lucknow, Uttar Pradesh, India

Corresponding Author: Pooja Ramakant, Associate Professor Department of Endocrine and Breast Surgery, King Georges' Medical University, Lucknow, Uttar Pradesh, India, Phone: +919791507780, e-mail: poojaramakant@rediffmail.com
}

\section{Source of support: Nil}

Conflict of interest: None

\section{INTRODUCTION}

We discuss patient management taking into consideration the detailed surgical techniques to provide a road map for doing difficult neck dissections. A 48-year-old female patient presented to our outpatient clinic with history of finishing last fractions of radiation therapy for carcinoma cervix and having noticed neck swelling for 1 week duration. She had no other comorbidities. On examination, she had hard goiter with restricted mobility and around $4 \mathrm{~cm}$ in diameter nodules with multiple enlarged nodes left level 2 to 5 . Her investigations done included fine needle aspiration cytology positive for malignancy and metastases in lymph nodes but needed more tissue for characterization of the malignancy type, core tissue biopsy mentioned differential diagnosis of ? poorly differentiated thyroid cancer/? Secondaries from carcinoma cervix, computed tomography scan of neck showed thyroid multinodular mass and multiple bulky nodal mass infiltrating esophagus and also encasing the carotid artery and internal jugular vein (IJV) on left side (Figs 1A and B), positron emission tomography (PET) scan done showed FDG-avid lesion in thyroid (SUV 10.2), left cervical lymph nodes (SUV 8.3), solitary tiny nodule in lung (SUV 2) and sclerotic changes in bone (SUV 9.2) both suspicious for metastases. Patient developed severe pain in neck over left level 2 nodes radiating to left ear and lower skull base for which she needed morphine supplementations and ryles tube feeding as had severe dysphagia. She was planned for early surgery in view of rapid progression of disease and to palliate her from severe unbearable neck pain.

\section{MATERIALS AND METHODS}

We describe surgical techniques of doing difficult neck dissections in patient with thyroid cancer with bulky nodes encasing the major vessels and compressing nerves causing severe neck pain. Kocher's incision was planned and we gave wide incision extending from one sternocleidomastoid (SCM) muscle to the other to have 


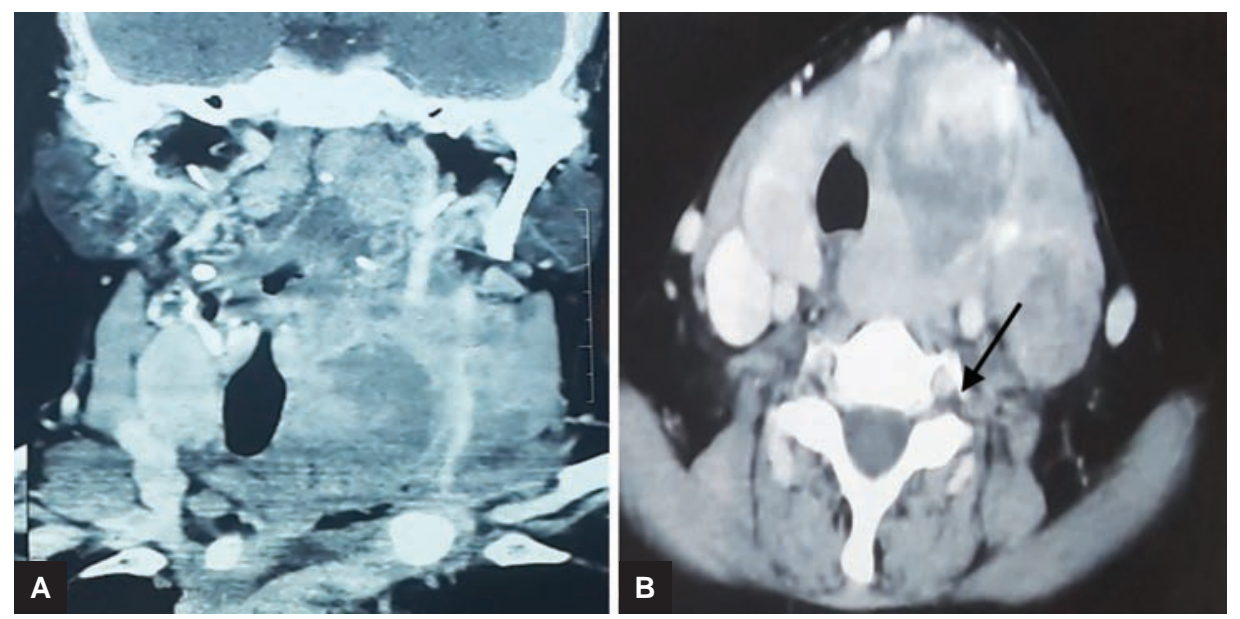

Figs $1 \mathrm{~A}$ and $\mathrm{B}$ : Computed tomography scan neck the thyroid nodules and encased carotid sheath by bulky left side nodal mass

good exposure as we anticipated difficult surgery. Subplatysmal flaps raised and both SCMs separated from strap muscles. The strap muscles were divided in view of being infiltrated by the thyroid nodules and take off with the total thyroidectomy and central compartment neck dissection specimen in toto. The right recurrent laryngeal nerve (RLN) was saved but left RLN could not be visualized due to large nodal mass and left lobe thyroid conglomerate mass adherent to underneath structures.

There were large, hard, adherent and at few areas friable nodal masses encasing the entire carotid sheath. At no place in the middle and lower part of neck, we could identify the IJV and common carotid artery (CCA). We took control of IJV at lower neck end so that, if it gets injured/opened up, we can ligate it and avoid any embolism. Then we tried to trace IJV at extreme upper part of neck and after identifying it, we inserted a small artery forceps and starting splaying the nodal mass and created plane just above the IJV dividing nodal mass medial and lateral to it (Fig. 2). It was like opening a book

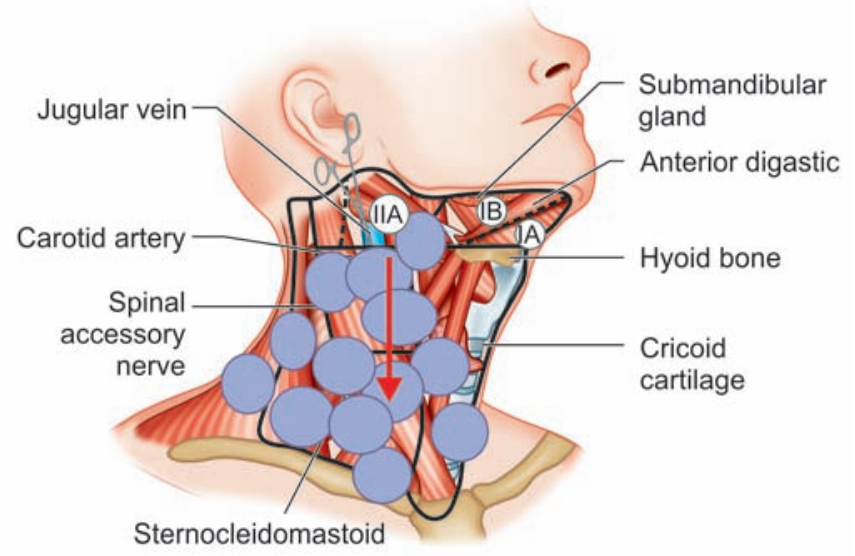

Fig. 2: Surgical technique of splaying of nodes medial and lateral to carotid sheath

and we could trace whole tract the entire IJV and CCA by slowly dissecting over the adventitia of major vessels with a fine small artery forceps working just above the carotid sheath (Figs 3A and B). We could dissect off all

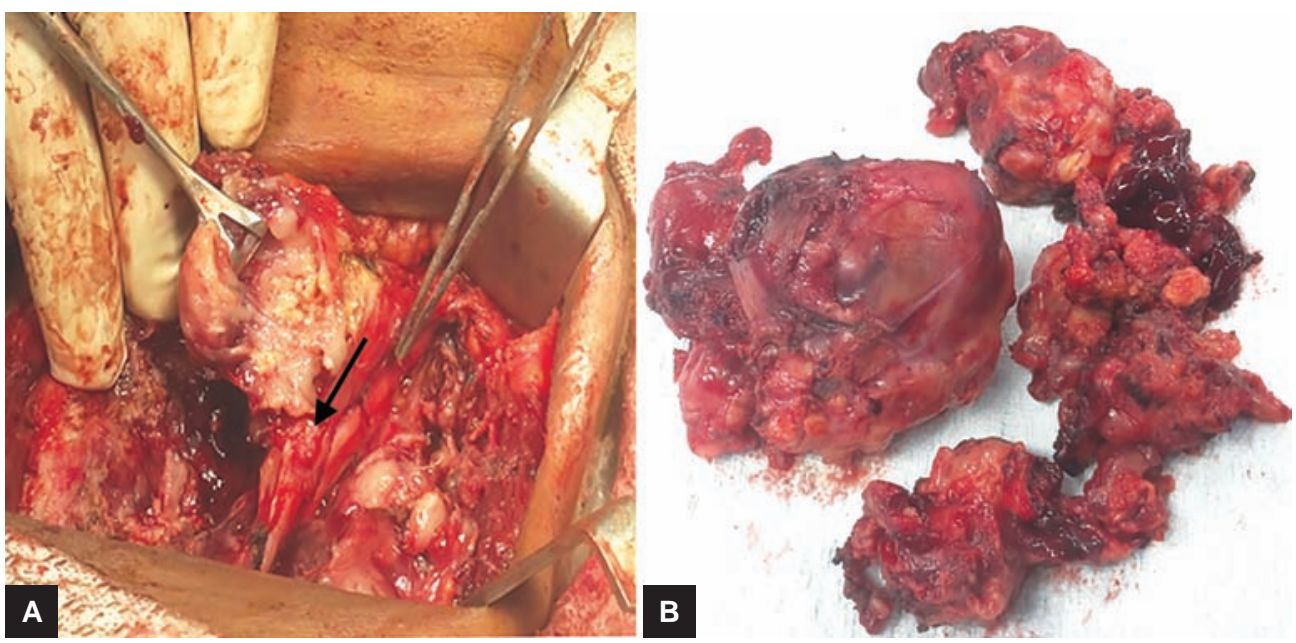

Figs 3 A and B: Intraoperative picture showing the entire carotid sheath and nodal masses medial and lateral to it and the entire thyroidectomy with central compartment and left modified radical neck dissection specimen 
nodal mass medial and lateral to it. At lower end of neck, we could identify and save thoracic duct also. The nodal mass was extending below clavicles but could be taken out with gentle firm traction. We used vessel sealer along with cautery (monopolar and bipolar) for good hemostasis. The level 2 node was adherent to Spinal Accessory nerve and shaved off from it by meticulous dissection.

Patient was extubated well and was kept on oxygen support for 6 hours. She had mild hypocalcemia in postoperative period, which was managed with oral calcium supplements. She had no voice change or any nerve injuries.

\section{DISCUSSION}

In patients with thyroid cancer, regional nodal metastases happen 30 to $80 \%$ of the times as reported in the literature. ${ }^{1}$ In difficult neck dissections, we either sacrifice major structures leading to morbidity while doing radical resections or in trying to save important structures, we compromise in doing $\mathrm{R} 0$ resections. ${ }^{2}$ Completeness of resection is directly proportional to the outcome of the patient in terms of regional persistence/recurrence and also to avoid morbidity of reoperative surgeries. ${ }^{3}$ Selective berry picking of nodes is not recommended as that leads to higher recurrences. ${ }^{4}$ By learning fine details of techniques of doing meticulous neck dissections, we can minimize morbidity as well as ensure best cure rates by doing R0 resections ${ }^{5,6}$ In patients with thyroid cancer, recurrences happen in 20 to $30 \%$ of the patients and most recurrences are due to insufficient initial surgery. ${ }^{7}$

Hence, it is very important to understand finer details of doing neck dissections in thyroid cancer to achieve best possible cure rates.

\section{CONCLUSION}

We recommend doing complete neck dissections including level 2 to 5 nodes in locally advanced thyroid cancers by splaying of large nodal masses medial and lateral to carotid sheath in order to reduce persistence/recurrence of the disease and to improve clinical outcome.

\section{CLINICAL SIGNIFICANCE}

By complete neck dissections and saving vital structures, we can improve patient's clinical outcome and minimize morbidity and reoperative surgeries.

\section{ACKNOWLEDGMENT}

Authors would like to thank the Endocrine Surgery Office staff for the technical helps.

\section{REFERENCES}

1. Dimov R, Deenichin G, Damianliev R, Uchikov A, Stefanov $\mathrm{Ch}$, Batashki I. Safety and efficacy of modified radical lymph nodes dissection in patients with papillary thyroid cancer (PTC) and clinically manifested lymph metastasis. Khirurgiia (Sofiia) 2006;(2):31-35.

2. Ardito G, Revelli L, Polistena A, Lucchini R, Giustozzi E, Guidi ML, Ardito F, Avenia N. Complications of Neck Dissections in Papillary Thyroid Carcinoma: A Modified Procedure to Reduce Parathyroid Morbidity. In Vivo 2016 May-Jun;30(3):303-308.

3. Javid M, Graham E, Malinowski J, Quinn CE, Carling T, Udelsman R, Callender GG. Dissection of Levels II Through V Is Required for Optimal Outcomes in Patients with Lateral Neck Lymph Node Metastasis from Papillary Thyroid Carcinoma. J Am Coll Surg 2016 Jun;222(6):1066-1073.

4. Pingpank JF Jr, Sasson AR, Hanlon AL, Friedman CD, Ridge JA. Tumor above the spinal accessory nerve in papillary thyroid cancer that involves lateral neck nodes: a common occurrence. Arch Otolaryngol Head Neck Surg 2002 Nov;128(11):1275-1278.

5. Sakorafas GH, Sampanis D, Safioleas M. Cervical lymph node dissection in papillary thyroid cancer: current trends, persisting controversies, and unclarified uncertainties. Surg Oncol 2010 Jun;19(2):e57-e70.

6. Caron NR, Clark OH. Papillary thyroid cancer: surgical management of lymph node metastases. Curr Treat Options Oncol. 2005 Jul;6(4):311-322.

7. Goyal RM, Jonklaas J, Burman KD. Management of recurrent cervical papillary thyroid cancer. Endocrinol Metab Clin North Am 2014 Jun;43(2):565-572. 